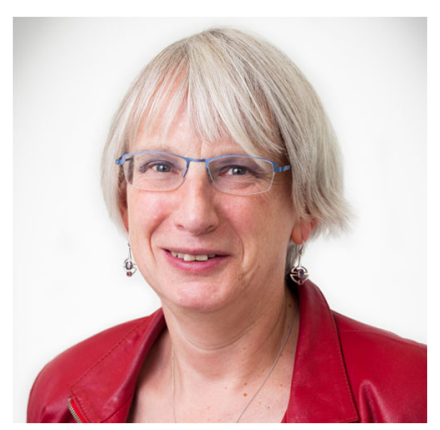

Competition for publication in Diabetologia continues to grow, and less than 20\% of papers are accepted. Of all the high-quality papers that appear in this month's issue I want to draw your attention to five articles that I think are particularly interesting. The articles are summarised here. Our publisher, Springer, has kindly made the full text of each of these papers freely available.

I hope you enjoy reading them!

Sally M. Marshall, Editor

\section{G protein-coupled receptors as new therapeutic targets for type 2 diabetes}

\section{Frank Reimann, Fiona M. Gribble}

G protein-coupled receptors (GPCRs) in the membrane of intestinal endocrine cells detect digested nutrients. In a review in this issue, Frank Reimann and Fiona Gribble discuss how GPCRs are the targets for existing glucoselowering therapies, as well as for a number of drugs in the pipeline. They explain how different GPCRs in gut endocrine cells, pancreatic islets and the brain are being targeted, with the aim of mimicking the postprandial state of enhanced insulin secretion and reduced appetite. Glucagon-like peptide-1 receptor agonists have highlighted the potential efficacy of targeting GPCRs for the treatment of diabetes, and are particularly popular because they exhibit glucose dependence at the level of the pancreatic beta cell and promote weight loss. By harnessing the tissue specificity and 'druggability' of GPCRs, it is hoped that new classes of diabetes therapies will emerge that target selected panels of tissues and exhibit superior side-effect profiles. This review is accompanied by a commentary by the Session Chair, Michael Nauck (DOI: 10.1007/s00125-015-3795-1).
Vaccinations and childhood type 1 diabetes mellitus: a meta-analysis of observational studies

Eileen Morgan, Sophia R. Halliday, Gemma R. Campbell, Chris R. Cardwell, Chris C. Patterson

There is continued debate about the environmental factors responsible for the increasing incidence of childhood type 1 diabetes, which, best-available evidence suggests, began in the middle of the last century. The fact that this increase coincides with the introduction of mass vaccination programmes in many countries has led to speculation that there may be a link. Although studies in the literature have generally provided little support for such an association, the power of many of these studies was low. In this issue, a systematic review and meta-analysis of observational studies by Morgan et al indicates that vaccination is not linked to any statistically significant increase in childhood diabetes risk. Although ongoing prospective studies of high-risk birth cohorts may add further evidence, the selective nature of these cohorts and the high vaccination rates in many populations mean that our metaanalysis may provide the most definitive answer to this issue. 
The impact of gender on the long-term morbidity and mortality of patients with type 2 diabetes receiving structured personal care: a 13 year follow-up study

Marlene Ø. Krag, Lotte Hasselbalch, Volkert Siersma, Anni B. S. Nielsen, Susanne Reventlow, Kirsti Malterud, Niels de Fine Olivarius

There is an increasing focus on personally tailored diabetes treatment, but it is widely unknown how gender impacts the effect of treatment. In this issue, Krag et al report that tailored diabetes treatment and individual goal setting (in collaboration with a general practitioner) significantly reduces mortality in women. However, the same effect is not seen in men. The authors suggest that tailored treatment could provide women with attention and support, thus providing an incentive for treatment adherence. Furthermore, women accept disease and implement disease management more easily, whereas men may feel challenged by diabetes, which demands daily consideration and lifestyle changes. The authors propose a need to further explore whether gender-specific treatment schemes could provide a similar reduction in mortality in men.

Effect of atorvastatin on glycaemia progression in patients with diabetes: an analysis from the Collaborative Atorvastatin in Diabetes Trial (CARDS)

Shona J. Livingstone, Helen C. Looker, Tahira Akbar, D. John Betteridge, Paul N. Durrington, Graham A. Hitman, H. Andrew W. Neil, John H. Fuller, Helen M. Colhoun

Statins are known to increase diabetes incidence, but information on the frequency, size and persistence of effects on glycaemia among individuals with established diabetes are lacking. In this issue, Livingstone et al present the results of an analysis of data from the Collaborative Atorvastatin Di- abetes Study (CARDS) randomised trial of atorvastatin $10 \mathrm{mg}$ in people with type 2 diabetes. According to their findings, the effect of atorvastatin $10 \mathrm{mg}$ on glycaemia progression among those with diabetes is common but very small, is apparent soon after statins are started but does not increase with duration of statin treatment and does not have an impact on the magnitude of the reduction in risk for cardiovascular disease with atorvastatin. These findings provide useful and reassuring data for balancing the risks and benefits of statin use in those with diabetes.

\section{Protein kinase STK25 controls lipid partitioning in hepatocytes and correlates with liver fat content in humans}

Manoj Amrutkar, Matthias Kern, Esther Nuñez-Durán, Marcus Ståhlman, Emmelie Cansby, Urszula Chursa, Elin Stenfeldt, Jan Borén, Matthias Blüher, Margit Mahlapuu

Type 2 diabetes is closely associated with pathological lipid accumulation in the liver, which is suggested to be involved in the development of insulin resistance. Serine/threonine protein kinase 25 (STK25) was recently identified as a regulator of liver steatosis, whole-body glucose and insulin homoeostasis in a mouse model system. In this issue, Amrutkar et al present the results of a study that investigated the role of STK25 in the regulation of lipid metabolism in human liver cells. Overexpression of STK25 in human hepatocytes enhanced lipid deposition by suppressing $\beta$-oxidation and triacylglycerol secretion while increasing lipid synthesis. Knockdown of STK25 in human hepatocytes produced the opposite effect. STK25 mRNA expression in human liver tissue samples was positively correlated with liver fat content. These data suggest that STK25 is a novel regulator of lipid partitioning in human liver and could be a potential drug target for the prevention/treatment of type 2 diabetes.

All text supplied by the authors. 